\title{
Study of Schottky barrier contact in hybrid CdSe Quantum dot organic solar cells
}

\author{
M. $\operatorname{Ramar}^{1}$, R. Manimozhi ${ }^{2}$, C. K. Suman ${ }^{1 *}$, R. Ahamad ${ }^{1}$, Ritu Srivastava $^{1}$, \\ ${ }^{l}$ CSIR-Network of Institutes for Solar Energy \\ ${ }^{1}$ CSIR - National Physical Laboratory, Dr. K. S. Krishnan Marg, New Delhi -110012, India. \\ ${ }^{2}$ J S S Academy of Technical Education, Noida, U.P - 201301, India. \\ E-mail: sumanck@mail.nplindia.ernet.in
}

\begin{abstract}
The hybrid organic solar cell devices are fabricated from P3HT:PCBM with Quantum dot CdSe materials.The Schottky barrier built-in potential of $0.45 \mathrm{~V}$ was calculated from $\mathrm{C}-\mathrm{V}$ measurements. The photo current $\left(J_{\text {Light }}-J_{\text {Dark }}\right)$ equals to zero at compensation voltage of $0.61 \mathrm{~V}$. The depletion width $(\mathrm{W})$ of an abrupt Schottky junction is calculated using carrier density. The Cole - Cole plot of the device is also determined from different bias voltage in the range of $10-1 \mathrm{M} \mathrm{Hz}$. The device can be modeled as the combination of RC parallel circuit.
\end{abstract}

Index Terms - Schottky barrier, compensation voltage.

\section{INTRODUCTION}

Hybrid organic solar cells (HOSCs) based on conjugated polymer, fullerene, quantum dot, nanowires and quantum tetrapods with bulk heterojunction (BHJ) are a good candidate for renewable energy resources because of their advantage of light-weight, flexibility, low-cost, and simple fabrication for large area processing. The high mobility and light absorption coefficient in visible region of polythiophenes make it one of the most important electron donor and fullerene composites, poly(3-hexylthiophene) (P3HT):[6,6]phenyl C61 butyric acid methyl ester (PCBM) blend are most widely used as an active layer and have reported remarkably improved performances of BHJ solar cells up to $5 \%$ of power conversion efficiency in single device [1].

A thorough understanding of the device physics of polymer based hybrid nano solar cells is a crucial prerequisite for purposeful optimization of these devices and materials. CdSe quantum dots of appropriate size can have an absorption edge and emission peak anywhere in the visible spectrum. This led to CdSe nanoparticles to be used for optoelectronic devices. The study of capacitance/voltage (CV) measurements done on hybrid polythiophene:fullerene solar cells, there has been a recent debate about the correct band diagram of hybrid polymer:fullerene with nano materials bulk heterojunction cells and in particular about the relevance and magnitude of doping and contact barriers [2,3]. In this paper, the ternary solar cells were fabricated with P3HT, PCBM and CdSe. The Schotky contact for these devices have been studied using capacitance - voltage and impedance spectroscopy.

\section{EXPERIMENTAL DETAILS}

P3HT and PCBM (1:0.8 weight ratio) were separately dissolved in chlorobenzene solvent $(30 \mathrm{mg} / \mathrm{ml})$. Then $10 \%$ of $\mathrm{CdSe}$ QDs were added in P3HT:PCBM blends. Indium tin oxide (ITO) coated glass substrate was cleaned in ultrasonic bath with acetone, isopropyl alcohol and D.I. water for 10 min, respectively. PEDOT:PSS solution was spin coated onto ITO substrate then annealed at $120^{\circ} \mathrm{C}$ for $20 \mathrm{~min}$ in vacuum. Photoactive layer (P3HT:PCBM added CdSe QDs) was deposited on PEDOT:PSS layer by spin coating. Thermal preannealing was conducted at $120{ }^{\circ} \mathrm{C}$ for $10 \mathrm{~min}$ in vacuum. Then Al top electrode was deposited on photo active layer by thermal evaporation. We fabricated the final solar cells device as the structure of ITO/PEDOT:PSS/P3HT:PCBM:CdSe/Al as shown in Fig. 1.

\section{RESULTS \& DISCUSSIONS}

The energy level diagram of the hybrid cdse blended organic solar cell devices are shown in fig 1 . The efficient photo induced charge transfer generates positive charge on P3HT and a negative charge on CdSe and PCBM. The negative charge on CdSe is transferred to PCBM finally to the cathode. The absorption maximum of the active layer thin film is observed at a wavelength of $504 \mathrm{~nm}$.

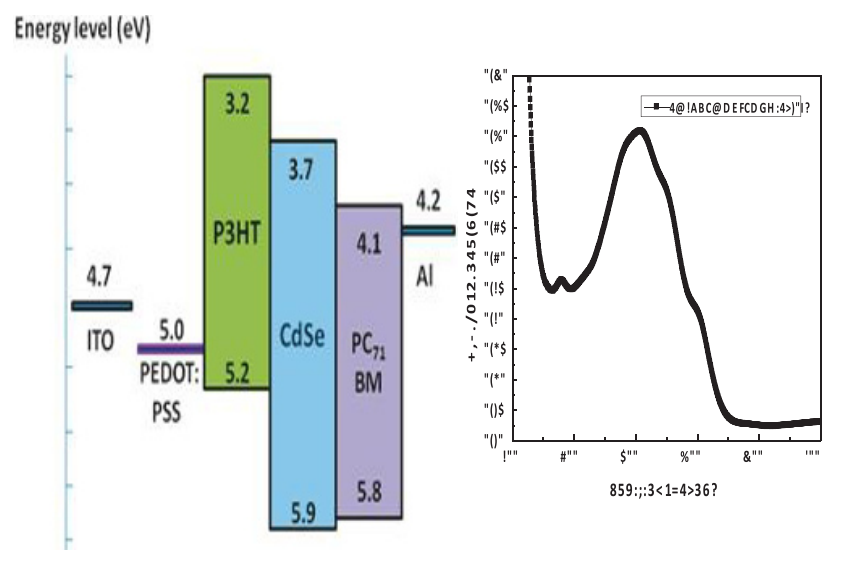

FIG.1. Schematic diagram of the energy level of ternary blend organic solar cells and absorption of the active layer thin film 


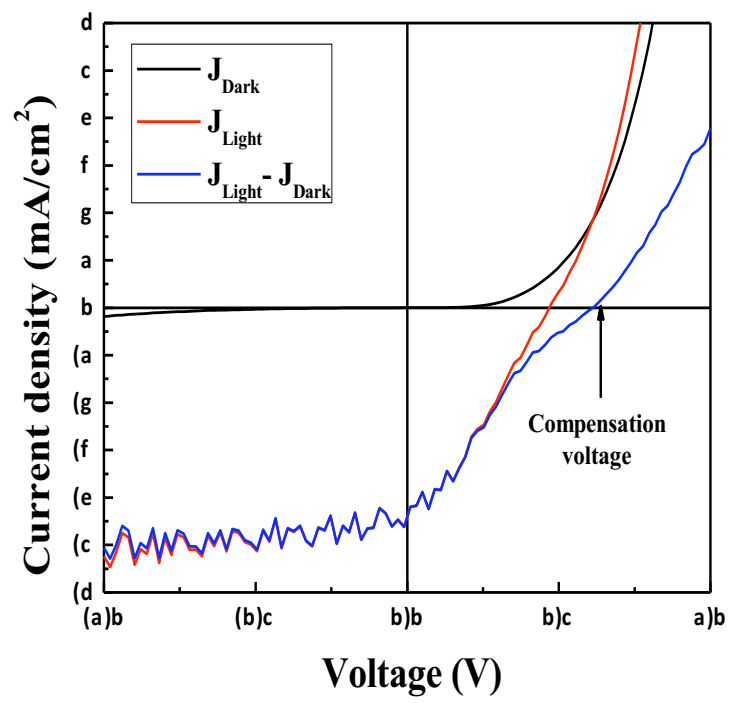

FIG. 2. $J-V$ characteristics of the device. The photocurrent

$\left(J_{\text {Light }}-J_{\text {Dark }}\right)$ equals zero at a compensation voltage of $0.61 \mathrm{~V}$

Figure 2 shows the current density Vs voltage in dark and under illumination. The dark J-V characteristics match with the diode having the threshold voltage $0.49 \mathrm{~V}$. The efficiency of solar cells is $\sim 0.9 \%$ and fill factor of 37 . The The built-in potential found by $C-V$ measurements is in good agreement with the calculated value of built in potential from $J-V$ plots. In a typical Schottky contact, the built-in potential is equivalent to the voltage at which the photocurrent $\left(J_{\text {Light }}{ }^{-}\right.$ $J_{\text {Dark }}$ ) becomes zero [4].

Fig. 3(a) shows the capacitance-voltage characteristics of the device measured with $\mathrm{AC}$ signal at $1000 \mathrm{~Hz}$. At room temperature the capacitance is practically independent of frequency and dc

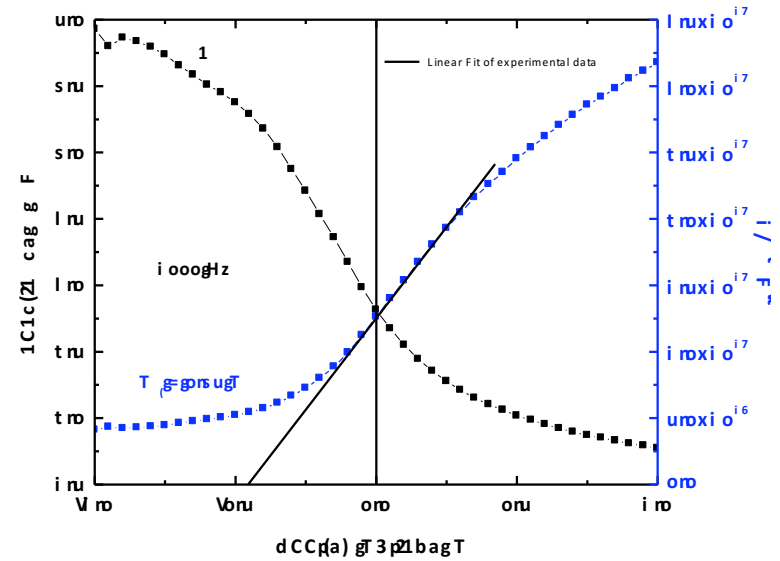

FIG.3. (a) $C-V$ characteristics of the device at $1000 \mathrm{~Hz}$ and (b) $1 / C^{2}$ Vs Applied Voltage Plot bias voltage. Fig. 3(b) shows the $1 / C^{2}-V$ characteristics. Analysis of the slope was performed by the usual standard Schottky relation

$$
\frac{d\left(\frac{1}{C^{2}}\right)}{d V}=\frac{-2}{N_{s} e \varepsilon A^{2}}
$$

Where, $N_{s}$ is the total charge (trapped plus ionized centers), $e$ the electronic charge, $\varepsilon$ the dielectric constant, and $A$ the area. The calculated value of $N_{s}$ is $1.59 \times 10^{21} / \mathrm{cm}^{3}$.

The depletion width $\mathrm{W}$ of an abrupt Schottky junction is calculated from Moot-Schottky equation (2). The depletion width $\mathrm{W}$ is $0.2701 \mu \mathrm{m}$.

$$
W_{d}=\left[\frac{2 \varepsilon \varepsilon\left(V_{b i}-V\right)}{q N_{A}}\right]^{1 / 2}
$$

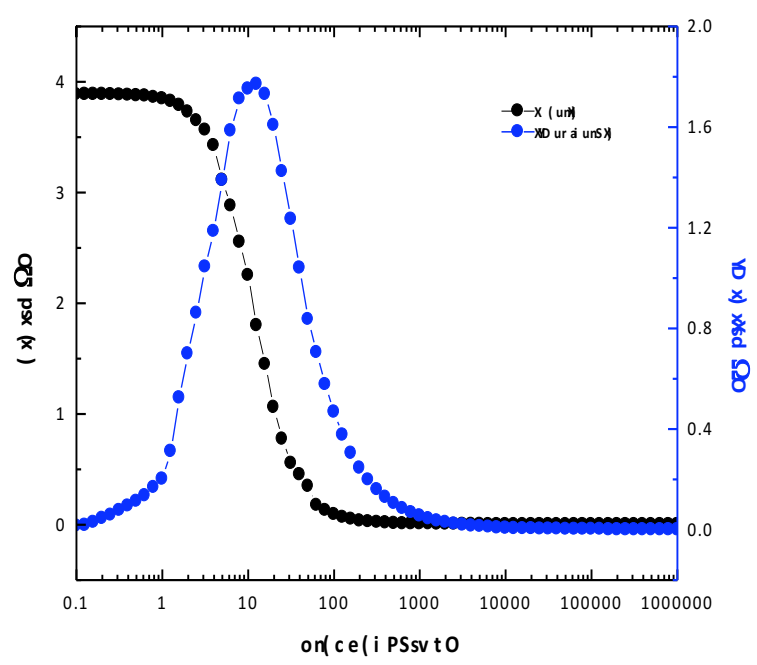

FIG.4. Impedance -Frequency plot at $0 \mathrm{~V}$ applied biasing

For complete analysis of impedance data Argand plot is needed which is the representation of impedance-frequency (Z-f) data on a complex plane, and it is useful to determine the equivalent circuit, since each circuit element responds differently to the frequency. The Argand representations of the data for the device show perfect semicircles in the complex plane, which corresponds to the response of a parallel resistance capacitance (RC) circuit. $[5,6]$ The total (complex) impedance of a parallel RC circuit with given resistance (R) and capacitance (C) is expressed as [7] 


$$
Z=\frac{1}{\frac{1}{R}+J \omega C}=Z_{R \mathrm{E}}+j R_{i m}
$$

$Z_{\mathrm{Re}}$ is the real part of the total impedance and $\mathrm{Z}_{\mathrm{Im}}$ is its imaginary part. Equations (3) can be reorganized to separate real and imaginary parts;

$$
\begin{aligned}
& Z_{R e}=\frac{R}{1+\omega^{2} R^{2} C^{2}} \\
& Z_{l m}=\frac{-\omega R^{2} C}{1+\omega^{2} R^{2} C^{2}}
\end{aligned}
$$

Two analyses are possible for obtaining separate $\mathrm{R}$ and $\mathrm{C}$ values from the $Z-f$ data in the Argand plot. First, when $\omega \rightarrow$ $0, Z_{R e}$ approaches $R$ and $Z_{I m}$ goes to zero according to Eq. (4). This means that the resistance $\mathrm{R}$ corresponds to the lowfrequency intercept of the curve with Real $(Z)$ axis. Next, the characteristic frequency $\omega_{c}$ at which $Z_{\mathrm{Im}}$ is minimum gives the $\mathrm{RC}$ time constant of the parallel RC circuit. By combining these two methods, $\mathrm{R}$ and $\mathrm{C}$ are independently accessible. In practice, $\omega_{\mathrm{c}}$ is obtained by nullifying the first derivative of $\mathrm{Z}_{\mathrm{Im}}$;

$$
\begin{aligned}
& \left.\frac{d Z_{I m}}{d(\omega)}\right|_{\omega=\omega}=0 \\
& \omega_{c}=2 \pi f_{c}=\frac{1}{R C}
\end{aligned}
$$

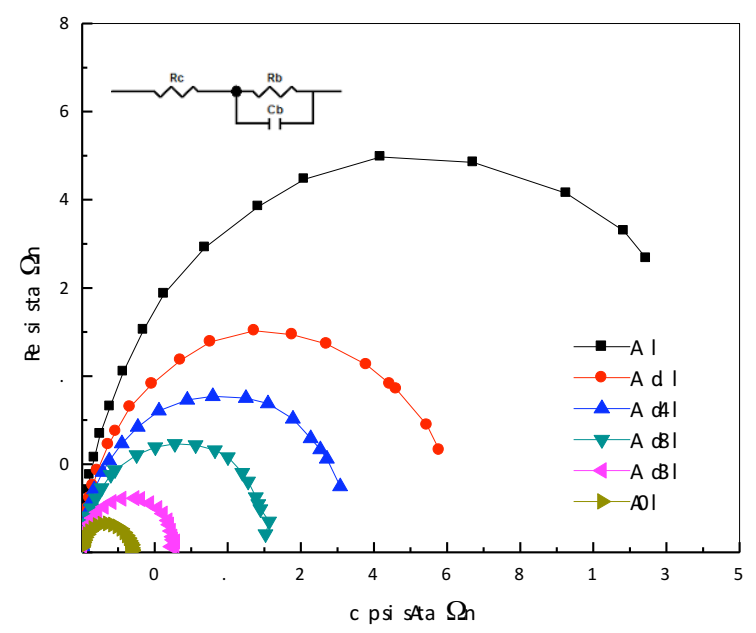

Fig. 5 Cole-Cole plot of the device at different biasing voltage
As shown by Eq. (5), the calculated value of $\omega_{c}$ (for the minimum $Z_{\mathrm{Im}}$ ), is the reciprocal of RC time constant, and then $\mathrm{f}_{\mathrm{c}}$ (in measurable "ordinary" frequency) is easily obtained. As one of the circuit parameters (namely, R) depends on the applied voltage,

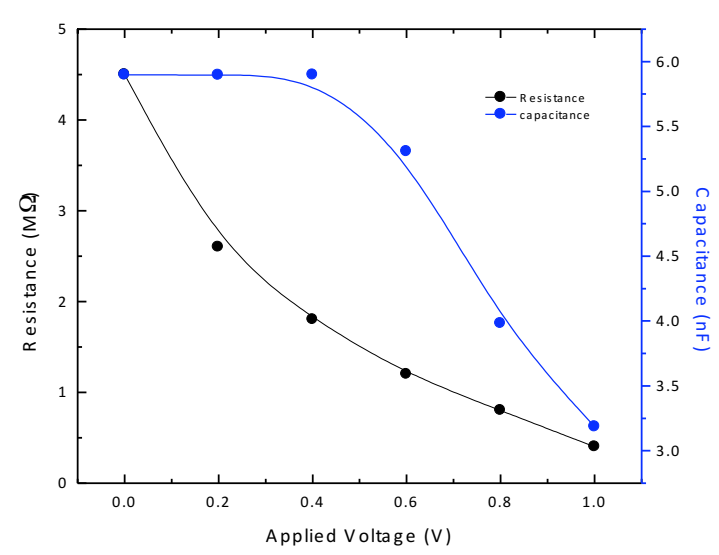

Fig. 6 Bulk resistance and capacitance as a function of applied voltage

Fig. 4. Z-f data were fitted to a simple parallel RC circuit using the software ZView. By comparison with other reports on the impedance analysis of organic diodes,[810] our data offer two distinctive features. First, we can neglect the contact resistance $\left(R_{c}\right)$ at the injecting contact (ITO_PEDOT:PSS interface), which often appears as a series element to the bulk circuit. The contact resistance might be negligibly small compared with the resistance of a thick, low mobility active materials bulk film and the realization of a lowresistance, high quality contact is attributed to the fact that the diode was fabricated under a controlled oxygenfree ambient. Next, a single capacitance element is observed and can be directly related to the geometrical capacitance of the diode as defined by the overlapped area of two electrodes. Both circuit parameters, resistance and capacitance, were extracted from the fitting process from the figure 5 too. The cole-cole plots of the device at different bias voltage in dark are shown in figure 5 . The semicircle reduces as the biasing voltage increases which is clearly indicating the decrease in bulk resistance with applied bias voltage. The equivalent circuit is shown in inset of figure 5 representing as the combination of parallel resistancecapacitance(R-C) circuits. Figure 6 shows the variation of the resistance and capacitance with applied voltage (V). The bulk resistance decreases with $\mathrm{V}$ because of increasing injected charge density in the semiconductor. The capacitance is practically constant in the forward bias regime upto the built in potential region. We attribute this small increase of the capacitance in the forward-bias regime to a small quantity of trapped charges inside the semiconductor bulk (no charges exist in the semiconductor bulk under reverse-bias). 


\section{CONCLUSIONS}

The Schottky contact is formed at cathode (Aluminium) side of the device. The built-in potential for the contact is 0.45 $\mathrm{V}$ and the compensation voltage is $0.61 \mathrm{~V}$. The efficiency of the device is $0.9 \%$ and the fill factor is $\sim 37$. The carrier density at the edge of the depletion layer is $1.59 \times 10^{21} / \mathrm{cm}^{3}$ and the depletion width of the Schottky abrupt junction is found to be $0.2701 \mu \mathrm{m}$. Impedance analysis at all regimes shows a single capacitance element corresponding to the geometrical capacitance. The device can be well modeled as the R-C circuits.

\section{REFERENCES}

[1] W. Ma, C. Yang, X. Gong, K. Lee, A. J. Heeger,thermally stable, efficient polymer solar cells with nanoscale control of the interpenetrating network morphology. Adv. Funct. Mater. 15 1617(2005).

[2] Fabregat-Santiago, F.; Garcia-Belmonte, G.; Mora-Sero, I.; Bisquert, $J$. Phys. Chem. Chem. Phys., 13, 9083-9118 (2011).
[3] Bisquert, J.; Garcia-Belmonte, G. J. Phys. Chem. Lett., 2, 1950-1964 (2011).

[4] Malliaras, G. G.; Salem, J. R.; Brock, P.J.; Scott, J. C. J. Appl. Phys., 84 (3), 1583-1587(1998)

[5] B. N. Pal, J. Sun, B. J. Jung, E. Choi, A. G. Andreou, and H. E. Katz, Adv. Mater. 20, 1023 (2008).

[6] Y. Li, J. Gao, D. Wang, G. Yu, Y. Gao, and A. J. Heeger, Synth. Met. 97, 19(1998).

[7] C. K. Alexander and M. N. O. Sadiku, Fundamentals of Electric Circuits, 2nd ed. (McGraw-Hill, New York, (2004).

[8] D. Braga, M. Campione, A. Borghesi, and G. Horowitz, Adv. Mater. 22, $424(2010)$.

[9] V. S. Reddy, S. Das, S. K. Ray, and A. Dhar, J. Phys. D: Appl. Phys. 40, 7687 (2007).

[10] C.-C. Chen, B.-C. Huang, M.-S. Lin, Y.-J. Lu, T.-Y. Cho, C.-H. Chang, K.- C. Tien, S.-H. Liu, T.-H. Ke, and C.-C. Wu, Org. Electron. 11, 1901 (2010). 